Background When sexual partners have risky context characteristics (i.e., partner drinking alcohol within two hours before sex, $\geq 3$ age discordant, or met in public), adolescents are particularly vulnerable to having unprotected sex and acquiring sexually transmitted diseases. Based on social cognitive theory, we assessed the influence of adolescents' alcohol use; their friends' ages and alcohol use; and parental monitoring on adolescent sexual partner selection to identify potent predictive factors.

Methods Data were from an urban cohort of youth participating in the Project Northland Chicago group-randomised, alcohol preventive intervention trial. We used ordinal logistic regression to analyse the predictive effect of $8^{\text {th }}$ grade self, peers, and parents factors on a sexual partner context risk score of 0 to 3 with 1 point for each risk characteristic of 17-18 year old adolescents' most recent sex partner. We adjusted analyses for sexual partner relationship characteristics (casual or unexpected).

Results Women were more likely to choose risky context partners at ages $17-18$ years old if in $8^{\text {th }}$ grade they had older friends [Odds Ratio $(\mathrm{OR})=1.5,95 \%$ Confidence Interval $(\mathrm{CI})=1.1$ to 2.1$]$ or reported risky alcohol use behaviours $(\mathrm{OR}=1.6,95 \% \mathrm{CI}=1.2$ to 1.9). Men were more likely to choose risky context partners at ages 17-18 years old if in $8^{\text {th }}$ grade their friends were drinking alcohol $(\mathrm{OR}=1.3,95 \% \mathrm{CI}=1.0$ to 1.7$)$. Parental monitoring did not influence partner selection.

Conclusions Peers and alcohol use influence adolescents' selection of risky context partners. For alcohol, self-use appears more important among women, and friends' use appears more important among men. Interventions to reduce sexual risk-taking and risky partner selection among adolescents should target friends and alcohol use.

\section{P4.076 SELF-STIGMA AND UNSAFE SEX AMONG MEXICAN MEN WHO HAVE SEX WITH MEN OF THE CONDESA SPECIALIZED CLINIC}

doi:10.1136/sextrans-2013-051184.0974

${ }^{1} \mathrm{H}$ Vega-Ramirez, ${ }^{2} \mathrm{C}$ Lara-Muñoz, ${ }^{1} \mathrm{D}$ Ferreyra, ${ }^{1} \mathrm{G}$ Hernandez-Heimpel, ${ }^{1} \mathrm{~J}$ Cruz-Islas, ${ }^{1} \mathrm{~V}$ Rodriguez-Perez. ' ${ }^{2}$ Condesa Specialized Clinic, Mexico City, Mexico; ${ }^{2}$ School of medicine BUAP, UNAM, Mexico City, Mexico

Background In Mexico, the HIV estimated prevalence among men who have sex with men (MSM) is $17 \%$, being the sexual transmission the most important via (90\%). In other countries, studies report that almost $43 \%$ of MSM with HIV (MSM-H) have unsafe sex. Use of alcohol, substances, misinformation of HIV transmission, perceived low-risk of infection, self-stigma, and others, are related to this type of sexual behaviour. Self-stigma in MSM-H who have unsafe sex has been poorly studied, and had contradictory results.

Methods Over 2012, after the ethical requirements, we asked MSM-H about unsafe sex with the Behavioral Surveillance Survey for MSM of 2006, and self-stigma with the HIV/AIDS Stigma InstrumentPLWA (HASI-P). This study was conducted at the Condesa Specialized Clinic in Mexico City, which is the largest Latin American clinic for people with HIV. We determinate unsafe sex dichotomously if the participant had not used condom, or had used non-water soluble lubricants during anal sex. We observed that self-stigma score had a non-normal distribution, so we used the Wilcoxon-MannWhitney test to compare it between the two groups.

Results The total sample was $200 \mathrm{MSM}-\mathrm{H}$, and the mean age was 33.6 (S.D., 8.3) years old, and the level of education was 12.9 (S.D., 3.1 ) years (high-school equivalent). The $52.5 \%$ of the sample had unsafe sex and the median score of self-stigma was 6 (range: 0-28) points. After comparative analysis we didn't find any significant differences of self-stigma between the two groups $(p=0.23)$.

Conclusion The self-stigma among MSM-H is not a factor related to unsafe sex, because this behaviour involves other social factors that must be deeply studied, particularly in this population. We have to include in future studies factors such as impulsivity, mental disorders, use of alcohol or substances, or others aspects that could probably be related to this behaviour.

\section{P4.077 SEXUAL AND PHYSICAL VIOLENCE AGAINST SEX WORKERS: A QUALITATIVE SURVEY TO EXPLORE EXPERIENCES OF VIOLENCE PERPETRATED BY POLICE AMONG SEX WORKERS IN VICTORIA FALLS, ZIMBABWE, 2012}

doi:10.1136/sextrans-2013-051184.0975

H M Ndondo, S Maseko, S Ndlovu. Sexual Rights Centre, Bulawayo, Zimbabwe

Background Few studies in Africa provide detailed descriptions of the vulnerabilities of sex workers (SW) to sexual and physical violence perpetrated by police, and how this impacts on their HIV risk. This qualitative study documents SW's experiences of violence in Victoria Falls, Zimbabwe.

Methods Thirty-one SW, aged 18 years and above were recruited through peer sex workers to participate in three focus-group discussions. Focus group discussions were digitally recorded and transcribed verbatim. Using ATLAS. ti software, a set of a priori concept-driven codes were applied to the transcripts. The analysis concentrated on condom possession and use by sex workers, police interactions, experiences of violence, stigma and discrimination perpetrated by the police.

Results Analysis showed the pervasiveness of sexual and physical violence perpetrated by the police towards SW without impunity, commonly triggered by arbitrary arrests of sex workers for solicitation and loitering. Sex workers narrated how police would arrest them for possession of condoms, confiscate and destroy condoms, extort money and bribes from them, force them to have sex with them, beat them with button sticks, drench them with cold water, detain them over nights in fenced enclosures and call them derogatory names. As a result some sex workers resorted to not carrying condoms or throwing away condoms to evade police harassment. Police were reported to profile sex workers and arrest them even when they were not doing sex work. Illegality of trading in sex in Zimbabwe was cited as one factor that underscored violence perpetrated by police.

Conclusions Addressing violence by the police and concomitant HIV risks and vulnerabilities faced by SW should be prioritised by the Zimbabwean government. This study indicates the need for legal reforms to decriminalise sex work and ensure that laws governing sex work promote the health and human rights of sex workers in Zimbabwe.

\section{P4.078 A LITERATURE REVIEW OF SEXTING ATTITUDES AND RISK FACTORS}

doi:10.1136/sextrans-2013-051184.0976

1.2L Lewis, ${ }^{3 R}$ Skinner, 'L Watchirs-Smith, ${ }^{4} \mathrm{~S}$ Cooper, ' $\mathrm{J}$ Kaldor, 'R Guy. ${ }^{1}$ The Kirby Institute, University of New South UK, Sydney, Australia; ${ }^{2}$ The University of Sydney, Sydney, Australia; ${ }^{3}$ Sydney University Discipline of Paediatrics and Child Health, The Children's Hospital at Westmead, Sydney, Australia; ${ }^{4}$ Sydney University Western Sydney Sexual Health Centre, Sydney, Australia

Background Sexting includes sending, receiving or forwarding sexually explicit material via mobile phone, and occurs in about a third of teenagers. Much of the available information on this relatively recent phenomenon comes from popular press. Given the legal and psychosocial consequences of sexting, it is important to understand why teenagers sext and the characteristics of young people involved in sexting.

Methods We conducted a structured literature review. PubMed, PsycINFO and Embase were searched to February 2013, using 
'sexting' as key term. We extracted data on; reasons for sexting, attitudes, and factors statistically associated with sexting.

Results Seven studies were included; most were cross-sectional, all were quantitative and conducted in the United States. Six studies assessed correlates of sexting in teenagers/young adults and found the following statistical associations; older adolescent, dating, sexually active, sexual risk behaviours, substance use, lower parental educational, peers sexting, and greater texting frequency. Girls were more likely to be senders, boys more likely to be receivers and to have asked someone to sext. Sexually active respondents were more likely to be both senders and receivers. Two studies explored attitudes about sexting finding those who sent pictures were more likely to consider sexting acceptable, over one third of non-sexters reported positive attitudes towards sexting, and most of those who sent pictures were bothered by having been asked to sext. Expecting serious legal consequences for getting caught sexting did not reduce reported sexting.

Conclusion Many young people don't perceive sexting negatively. Sexting may either be part of a cluster of risky sexual behaviours or in fact lead to sexual risk behaviour. Because of the cross-sectional nature of the studies, we were unable to determine causality. Additional research is needed to understand contexts in which sexting occurs, and motivations. Longitudinal designs are required to explore causality with sexual risk behaviour.

\section{P4.079 SEXUAL BEHAVIORS AND SAFETY STRATEGIES OF WOMEN WHO HAVE SEX WITH MEN AND WOMEN}

doi:10.1136/sextrans-2013-051184.0977

V R Schick, 'A Bell, 'C Neal, 'B Van Der Pol, 'B Dodge, 'L Bay-Cheng, ${ }^{3} \mathrm{~J} D$ Fortenberry. IIndiana University, Bloomington, IN, United States; ${ }^{2}$ University at Buffalo, Buffalo, NY, United States; Indiana University, Indianapolis, IN, United States

Background Women who have sex with women and men (WSWM) are at an increased risk for STI. Yet, knowledge regarding the behaviours associated with infection remains limited, with most studies focused on the experiences of women who have sex with only men or women. The diversity of sexual behaviours WSWM engage in may be limited by comparing WSWM to other groups. Instead, focusing on their experiences exclusively may provide a more comprehensive understanding of the sexual lives of WSWM.

Methods Local (Indianapolis, IN, US) women who had engaged in recent genital contact with a male and female partner were invited to complete an on-line survey followed by an interview. Participants were asked to indicate the most recent time they had participated in a variety of behaviours with a male and/or female partner.

Results Eighty participants ranging in age from 18 to $51(M=26.74$, $\mathrm{SD}=7.97)$ completed the survey. The most commonly reported sexual behaviours were similar for male and female partners, including kissing, cuddling, external genital rubbing, vaginal fingering, cunnilingus/fellatio and penile-vaginal intercourse. While less commonly reported, a sizable minority of participants reported vaginal fisting, anal fingering and analingus. Toy use was reported by the majority of the participants with vibrator use reported as the most commonly used toy. Approximately $75 \%$ of participants indicated sexual behaviour with more than one person at one time. The percentage of participants who reported barrier use varied by behaviour and partner gender with the lowest percentage of participants reporting use during oral sex or genital-on-genital rubbing with a female partner (>90\% never) and the highest percentage reporting use during penile-vaginal intercourse ( $>25 \%$ always).

Conclusion Participants reported engaging in a variety of sexual behaviours that may facilitate STI transmission. Further knowledge about the types of behaviours WSWM engage in may help inform risk reduction strategies.

\section{P4.080 UNDERSTANDING THE INFLUENCE OF INDIVIDUAL AND PARTNER-SPECIFIC SEXUAL SEALTH ON SEXUAL RISK BEHAVIOUR AMONG ADOLESCENT WOMEN}

doi:10.1136/sextrans-2013-051184.0978

D J Hensel, J Fortenberry. Indiana University School of Medicine, Indianapolis, IN, United States

Background Sexual health identifies both individual and partnerspecific factors as important elements in public health approaches to STI prevention among adolescents, yet little empirical evidence links them to specific STI-related health outcomes.

Method Data were individual and partner-specific quarterly interviews from a cohort of young women in Indianapolis $(\mathrm{N}=387$, 14-17 yrs.). Using an existing sexual health definition (WHO, 2002) we created two standardised, multi-item sexual health scales: individual sexual health (sexual positivity, absence of genital pain, family communication, family connexion) and partner-specific sexual health (relationship satisfaction, sexual satisfaction, condom use self-efficacy, pregnancy prevention attitudes, sexual communication, partner's connexion to family) (both $\alpha \geq 0.85$ ). Outcomes were: used a condom at last sex (no/yes), ratio of condom-protected coital events, any sexual coercion (no/yes), current number sex partners $(2+/ 1)$, future number of sex partners (next 90-days: $2+/ 1$ ). Analyses were multilevel logistic and linear regression (HLM, 7.0; all $p<0.05$ ), overall and by current number of sexual partners.

Results Individual $(\mathrm{OR}=1.22)$ and partner-specific $(\mathrm{OR}=1.87)$ sexual health predicted condom use at last sex; partner-specific sexual health predicted no sexual coercion $(O R=0.69)$, a higher ratio of condom-protected coital events $(b=0.12)$, as well as having one sexual partner currently and for the anticipated future. Higher partner-specific sexual health predicted condom use at last sex in currently single $(\mathrm{OR}=1.70)$ and in currently multiple partner relationships $(\mathrm{OR}=2.22)$, a higher ratio of condom protected coital events in currently single $(b=0.15)$ and in currently multiple partner relationships $(b=3.66)$, and absence of sexual coercion $(\mathrm{OR}=0.19)$ in currently multiple-partner relationships.

Conclusion Individual and partner-specific sexual health are separately linked to key STI-related public health indicators. These data suggest that different elements may require emphasis to more fully support effective sexual health approaches to reducing STI in adolescents.

\section{P4.081 STD RISK PERCEPTION AMONG HIGHER RISK UNIVERSITY STUDENTS IN HALIFAX, CANADA}

doi:10.1136/sextrans-2013-051184.0979

\section{B Langille, A Steenbeek. Dalhousie University, Halifax, NS, Canada}

Background Perceived risk is central to health behaviour theory, though little is known about what creates perceived risk of contracting STDs. We examined self-rated risk of STD in higher sexual risk (HSR) university students in Halifax, Canada, to determine factors associated with recognition of such risk status.

Methods Using an online survey, we asked university students about their perception of their STD risk (greatly/quite a lot at risk versus not very much/no risk), their sexual behaviours, chlamydia knowledge (CK), friends' more liberal attitudes to sexual risk-taking (FLASRT), depression, and personal factors. HSR was defined as having had both $\geq 2$ partners for vaginal sex in the past year and no condom use at last intercourse. Variables initially associated with perceived HSR $(p<0.10)$ were entered into a logistic regression model controlling for gender to determine which remained associated with perception of being at HSR.

Results The survey response rate was $32 \%(\mathrm{~N}=4490)$, and 526 were at HSR. Of those with 2-5 partners in the previous year, only $14 \%$ rated themselves as at HSR, while $43 \%$ of those with $\geq 6$ 